\title{
The Deformation Behavior of Oxygen-Modified
}

\section{Ti-29Nb-13Ta-4.6Zr(wt.\%)}

\author{
V. Khademi ${ }^{1,2}$, H. Liu ${ }^{3}$, M. Nakai ${ }^{4}$, M. Niinomi ${ }^{5}$, and C.J. Boehlert ${ }^{2 *}$ \\ 1. Department of Mechanical Engineering, Michigan State University, East Lansing, MI 48824, USA \\ 2 Department of Chemical Engineering and Materials Science, Michigan State University, East Lansing, MI 48824, USA \\ 3 Joining and Welding Research Institute, Osaka University, 11-1, Mihogaoka, Ibaraki, Osaka, 567-0047, Japan
}

4 Department of Mechanical Engineering, Faculty of Science and Engineering, Kindai University, Higashiosaka 577-8502,

Japan

5 Institute for Materials Research, Tohoku University, Sendai 980-8577, Japan

*Corresponding author email: boehlert@egr.msu.edu

\begin{abstract}
A series of Ti-29Nb-13Ta-4.6Zr(wt.\%) \{TNTZ $\}$ alloys containing either $0.1,0.3$ or 0.7 (wt.\%) oxygen (O) were roomtemperature tensile tested inside a scanning electron microscope to evaluate the effect of $\mathrm{O}$ on the deformation evolution. The deformation modes observed for TNTZ-0.1O, which exhibited the largest elongation-to-failure and lowest strength of all the alloys, were deformation-induced $\alpha "$-martensitic transformation, $\{332\}<113>$ twinning, and $<111>$ slip. For the other two alloys, $<111>$ slip was the dominant deformation mode, where TNTZ-0.7O exhibited more homogeneous and extensive slip, a higher frequency of cross slip, and a higher work-hardening rate, all of which contributed to both its strength and elongation-to-failure being greater than that for TNTZ-0.3O. TNTZ-0.3O exhibited the greatest tendency for cracking, which generally occurred on grain boundaries perpendicular to the tensile axis, leading to the lowest elongation-to-failure of all the alloys.
\end{abstract}

\section{Introduction}

(C) The Authors, published by EDP Sciences. This is an open access article distributed under the terms of the Creative Commons Attribution License 4.0 (http://creativecommons.org/licenses/by/4.0/). 
A novel $\beta$-type Ti alloy, Ti-29Nb-13Ta-4.6Zr(wt.\%) (hereafter abbreviated as TNTZ), composed of nontoxic and non-allergenic elements, has been developed for biomedical applications based on the d-electron design method [1,2]. This alloy exhibits a low Young's modulus (E 60 GPa), an intermediate ultimate tensile strength (UTS $\sim 510 \mathrm{MPa}$ ), and excellent corrosion resistance and biocompatibility [1]. Akahori et al. [3] performed various thermal-mechanical treatments on TNTZ to induce strengthening through $\alpha$ - and/or $\omega$-phase precipitation. However, these precipitates dramatically increased $\mathrm{E}$ (as the $\mathrm{E}$ values of the $\alpha$ and $\omega$ phases are higher than that of the $\beta$-phase) making it less attractive for load-bearing biomedical implant applications. Yilmazer et al. [4] were able to significantly increase the UTS of TNTZ through the microstructural refinement caused by high-pressure torsion. However, both of the abovementioned processes had a deleterious effect on the elongation-to-failure. Geng et al. [5], found that $\mathrm{O}$ addition, which has a significant solid-solution strengthening effect, is not costly as $\mathrm{O}$ is readily available, and poses no risk to the human body, could improve the UTS while not significantly affecting the $\mathrm{E}$ value. Increasing the $\mathrm{O}$ content of a metal generally results in increased strength at the expense of elongation-to-failure. However, an abnormal deformation behavior was observed in the case of O-modified TNTZ, where for small $\mathrm{O}$ additions, the strength increased and the $\mathrm{e}_{\mathrm{f}}$ decreased, but with a greater increase in $\mathrm{O}$ above $0.3 \mathrm{wt} \%$, both the strength and elongation-to-failure increased $[5,6]$. Because this behavior has yet to be fully explained, this study focused on characterizing the deformation behavior of O-modified TNTZ to shed light on this abnormal behavior.

\section{Materials and Experiments}

A series of TNTZ-xO (where $\mathrm{x}=0.1,0.3$, or $0.7 \mathrm{wt} . \%$ ) ingots were prepared by cold crucible levitation melting using as-received TNTZ rods and rutile $\left(\mathrm{TiO}_{2}, 99.9 \%\right.$ purity), which was added in the appropriate proportion in a high-purity argon atmosphere. The compositions of all the prepared ingots, which are listed in Table I, were determined by conventional chemical and gas analyses. The ingots were then homogenized at $1373 \mathrm{~K}$ for $6 \mathrm{~h}$ in an argon atmosphere and subsequently quenched in ice water. The ingots were then hot forged into cuboids and subsequently hot rolled into plates with an $83 \%$ reduction in thickness at 1273 $\mathrm{K}$ in an argon atmosphere and then cooled in air. The hot-rolled plates were then solution treated at $50 \mathrm{~K}$ above their respective b-transus temperature (1013 K for TNTZ-0.1O(wt.\%), $1093 \mathrm{~K}$ for TNTZ-0.3O(wt.\%), and $1243 \mathrm{~K}$ for TNTZ-0.7O(wt.\%) for $1 \mathrm{~h}$ 
in vacuum and subsequently quenched in ice water. It is noted that throughout the remainder of the test all alloy compositions are provided in weight percent un less otherwise noted.

Test samples were machined using electrical-discharge machining according to the geometry described in [7-11]. The samples were mechanically polished to a metallographic finish. The tests were performed at room temperature (RT) inside a Tescan Mira3 scanning electron microscope (SEM) and electron backscattered diffraction (EBSD) was performed on selected areas within the gage section of samples prior to and after the deformation according to the procedures outlined in [7-12]. The tests were paused several times for acquiring the SEM images. During these pauses, stress relaxation occurred. After each pause, loading was continued at the same displacement rate. Two tests were performed for TNTZ-0.1O, while one test was performed for both TNTZ-0.3O and TNTZ-0.7O. None of the samples were taken to failure. Secondary electron (SE) and backscatter electron (BSE) SEM photomicrographs were acquired before, during, and after the tensile experiments. The electron beam voltage used was $20 \mathrm{kV}$. For the provided SEM photomicrographs, the tensile axis was horizontal. The strains were estimated using the sample gage length $(10 \mathrm{~mm})$ and identifying the change in distance between surface fiducial marks, which in some cases were Vickers hardness indents. A slip trace analysis, previously described in [7-12], was used to identify the active slip deformation systems. Three families, namely $\{110\}<111>,\{112\}<111>$, and $\{123\}<111>$, with a total of 48 slip systems, were considered in this analysis. A threshold angle of $\pm 4^{\circ}$ was considered to determine the active slip systems and whenever multiple slip systems were possible, within this threshold angle, the slip system chosen was based on the highest Schmid factor.

Table I. The Measured Compositions in Weight Percent of the Studied Alloys

\begin{tabular}{|cccccccc|}
\hline Alloy & Ti & Nb & Ta & Zr & O & N \\
\hline TNTZ-0.10 & bal. & 29.3 & 13.2 & 4.67 & 0.091 & 0.0068 \\
TNTZ-0.30 & bal. & 27.7 & 13.5 & 4.65 & 0.28 & 0.006 \\
& & & & & & \\
TNTZ-0.70 & bal. & 28.8 & 12.2 & 3.72 & 0.724 & 0.0143 \\
\hline
\end{tabular}




\section{Results}

The average UTS values were $520 \mathrm{MPa}, 800 \mathrm{MPa}$, and $980 \mathrm{MPa}$ for TNTZ-0.1O, TNTZ-0.3O, and TNTZ-0.7O, respectively. Figure 1 provides sequential SE SEM photomicrographs and pretest and posttest EBSD inverse pole figure (IPF) maps for a TNTZ-0.1O sample. Figure 2 shows an EBSD IPF map from the gage section of this sample after $8.4 \%$ strain. Some of the grains exhibited $\{332\}<113>$ deformation twins, which were characterizd by a 50.5 degree misorientation along $<110>$. 166 of the 667 grains analyzed within the gage section of this sample exhibited such twins. This constituted $17 \%$ of the grains, while slip was observed in $20 \%$ of the grains. For example, see Figure 3 which illustrates the surface of the TNTZ-0.1O sample deformed to $5.8 \%$ strain. 

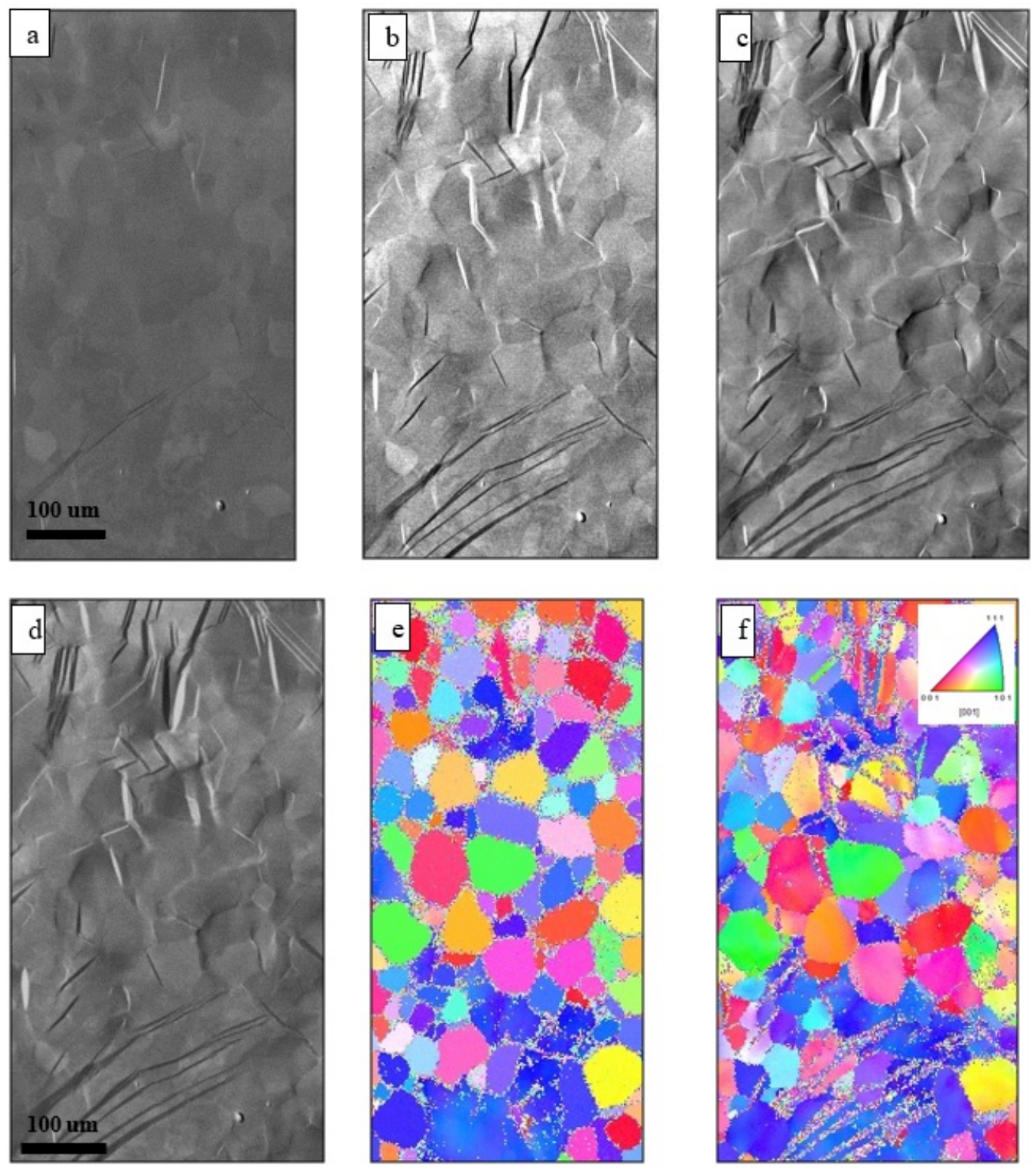

Figure 1. Sequential SE SEM photomicrographs acquired during the RT TNTZ-0.1O test at (a) $1.7 \%$ strain, (b) $3.1 \%$ strain, (c) $5.2 \%$ strain, and (d) $8.4 \%$ strain. Each of these images represents the same microstructural patch. (e) Pretest EBSD IPF map, and (f) Posttest EBSD IPF map. The tensile axis was horizontal. 


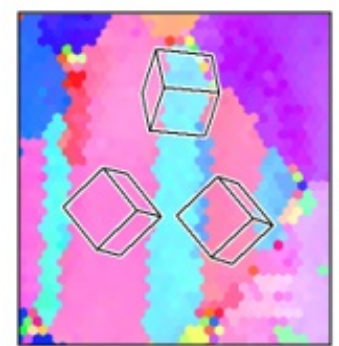

(b)

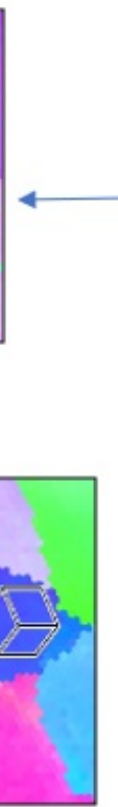

(c)

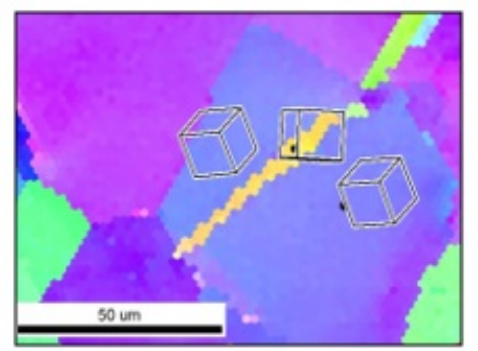

(d)

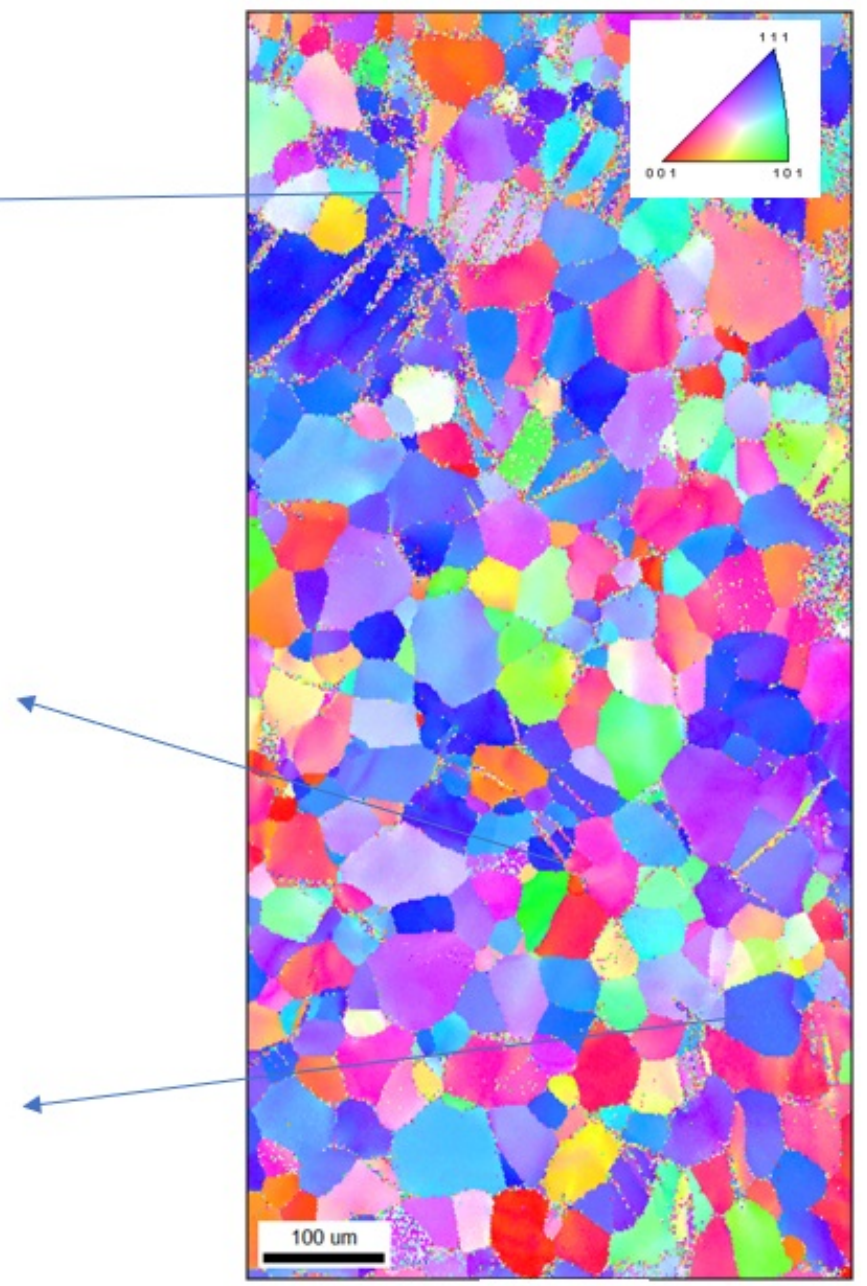

(a)

Figure 2. (a) EBSD IPF map from the gage section of the TNTZ-0.10 tensile tested sample after $8.4 \%$ strain. (b), (c), and (d) mechanical twins were observed in some grains. The misorientation angle between the $\{332\}<113>$ and the matrix was approximately 50.5 degrees along the $<110>\beta$ direction. 

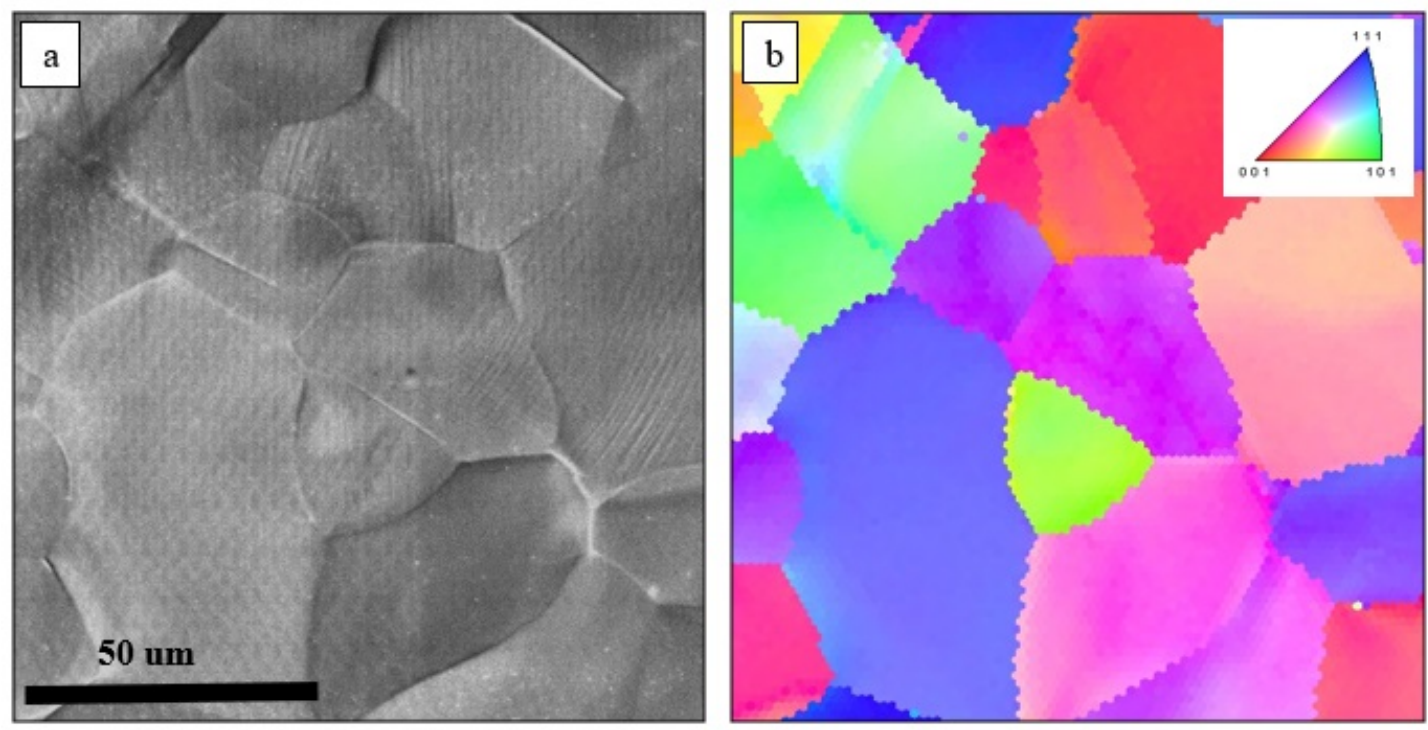

Figure 3. (a) SE SEM photomicrograph of a TNTZ-0.10 sample deformed to $5.8 \%$ strain. Fine slip traces are evident in some grains. (b) Posttest EBSD IPF map.

Figure 4 exhibits a SE SEM photomicrographs of the gage section of the TNTZ-0.3O sample after $4.6 \%$ strain. Figure 5 shows the pretest EBSD IPF map and corresponding sequential SE SEM photomicrographs for this sample. Cracks, which tended to form on grain boundaries oriented perpendicular to the tensile axis, were first observed at a plastic strain of $1.8 \%$. As shown in Figure $4 b$, only few slip traces were evident in the grains neighboring the cracks. No twins were observed in this sample. 

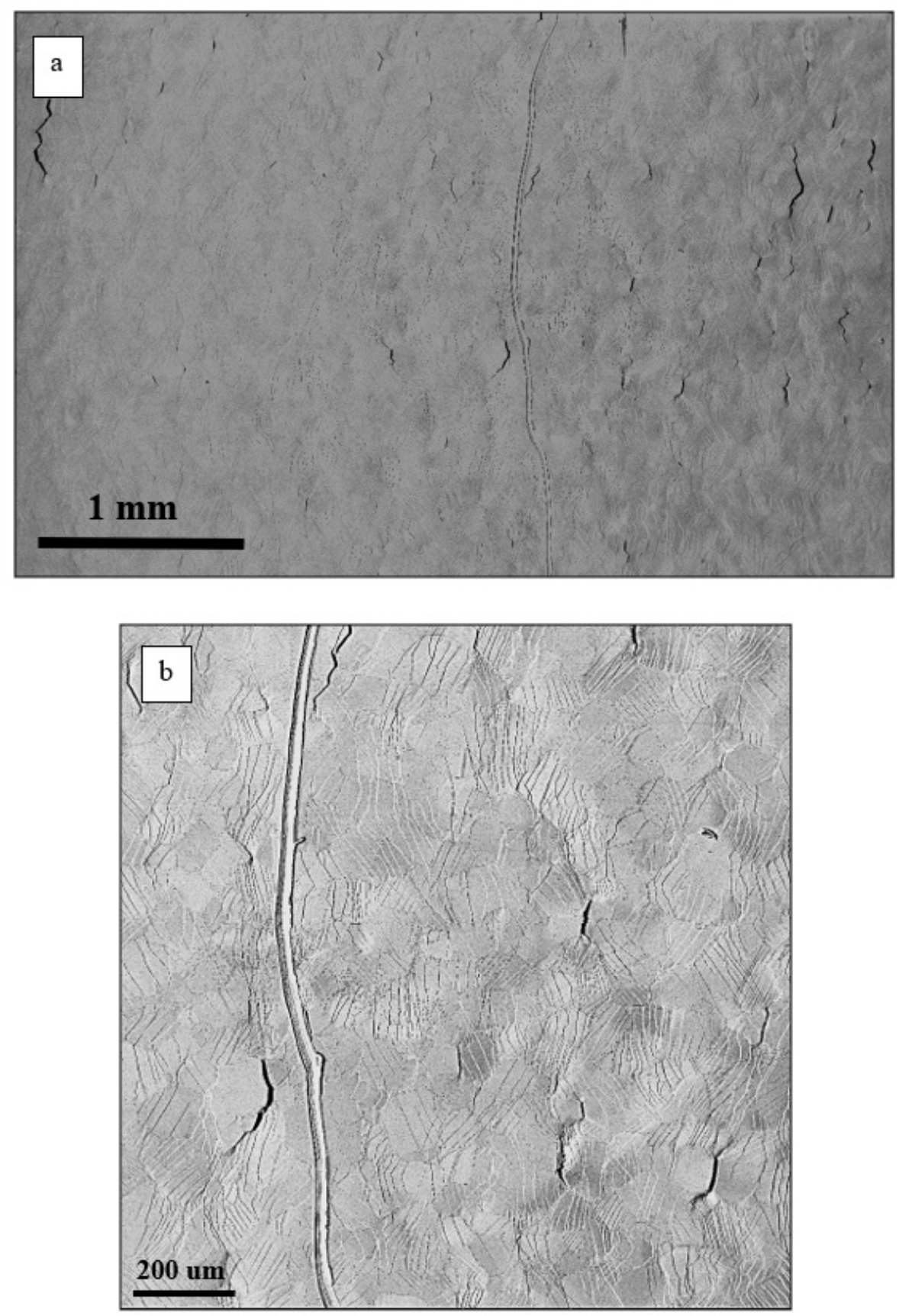
Figure 4. (a) Lower-magnification and (b) higher-magnification SE SEM photomicrographs of a deformed TNTZ-0.3O) sample deformed to $4.6 \%$ strain. Cracking, which tended to form on grain boundaries perpendicular to the loading direction, was observed throughout the gage section. The wavy vertical line was a imposed surface fiducial scratch.
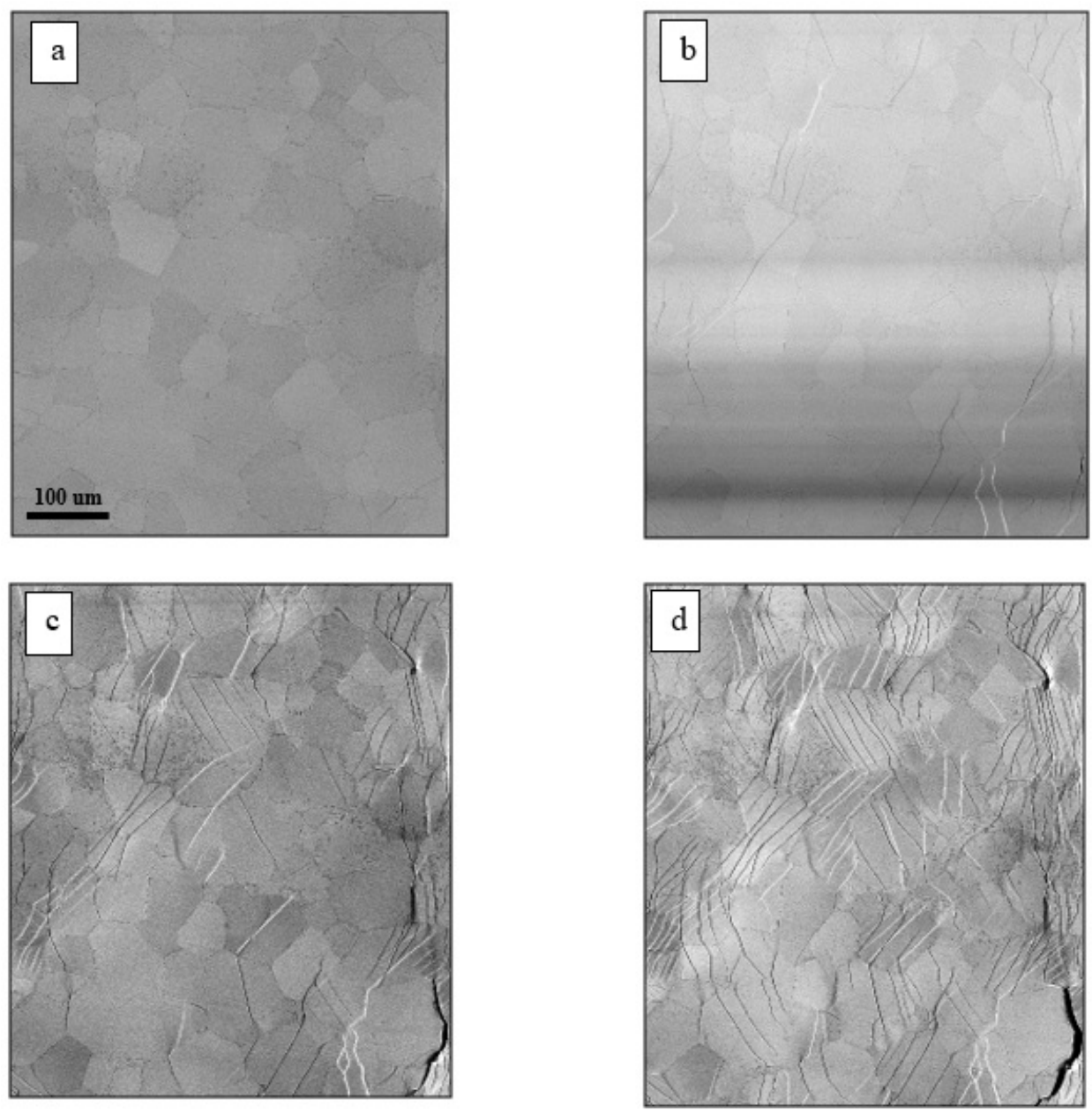

Figure 5. Sequential SE SEM photomicrographs acquired for a TNTZ-0.30 sample deformed to: (a) $0.9 \%$ strain, (b) $1.8 \%$ strain, (c) $2.9 \%$ strain, (d) $4.6 \%$ strain. Each of these images represents the same microstructural patch. 
Figure 6 shows the distribution of the different slip modes (identified after 2.9\% strain) versus the corresponding Schmid factor range. The slip trace analysis was performed on 215 grains (of which 177 grains exhibited at least one active slip system). The majority of the slip traces corresponded to $\{123\}<111>$ slip, while the minority of the slip traces corresponded to $\{110\}<111>$ slip.

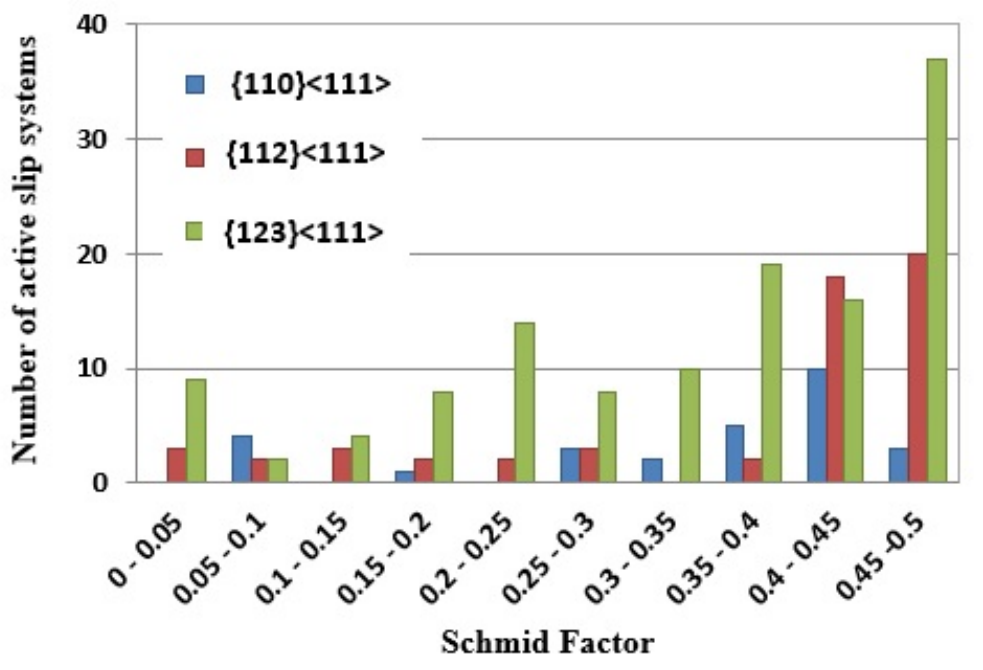

Figure 6. A histogram of Schmid factor distribution of slip systems for the TNTZ-0.3O sample after $\sim 2.9 \%$ strain.

Figure 7 exhibits a SE SEM photomicrograph of the gage section after $6.1 \%$ strain. Less cracking was evident in TNTZ-0.7O compared with TNTZ-0.3O. Figure 8 shows the pretest and posttest EBSD IPF map and corresponding sequential SE SEM photomicrographs for the same microstructure patch for TNTZ-0.7O. No twins were observed. 


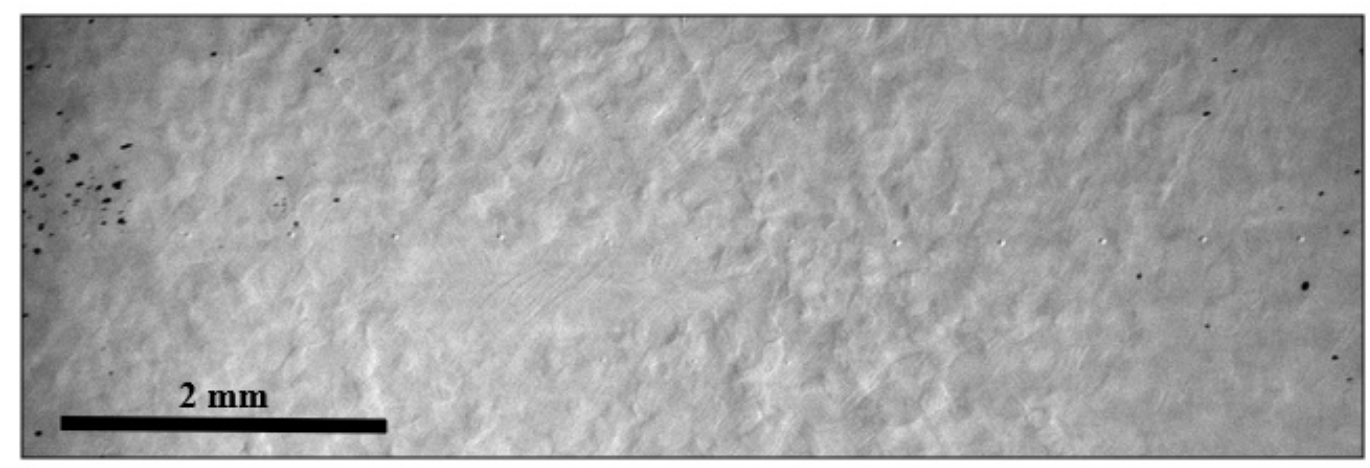

Figure 7. SE SEM photomicrograph of a TNTZ-0.7O sample deformed to 6.1\%. Uniform plastic strain was observed throughout the gage section. 

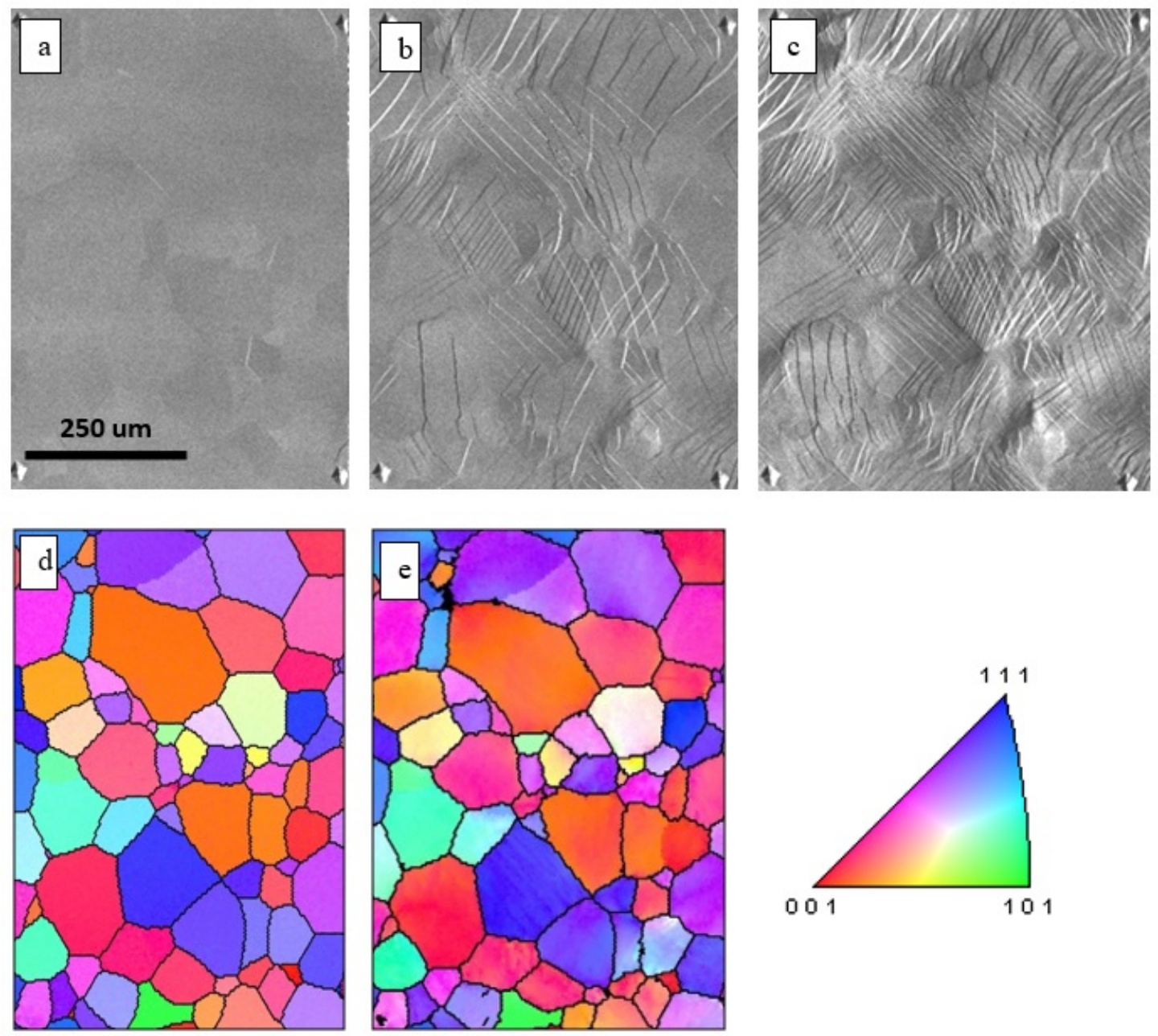

Figure 8. Sequential SE SEM photomicrographs acquired for TNTZ-0.7O deformed to: (a) $1 \%$, (b) $1.4 \%$, (c) $6.1 \%$ strain, and (d) Pretest EBSD IPF map, (e) posttest EBSD IPF map. Each of these images represents the same microstructural patch.

Figure 9 shows the distribution of the different slip modes identified at $6.1 \%$ strain versus the corresponding Schmid factor range for TNTZ-0.7O. The slip trace analysis was performed on 84 grains. The majority of the slip traces corresponded to $\{123\}<111>$ slip, while the minority of the slip traces corresponded to $\{110\}<111>$ slip. 


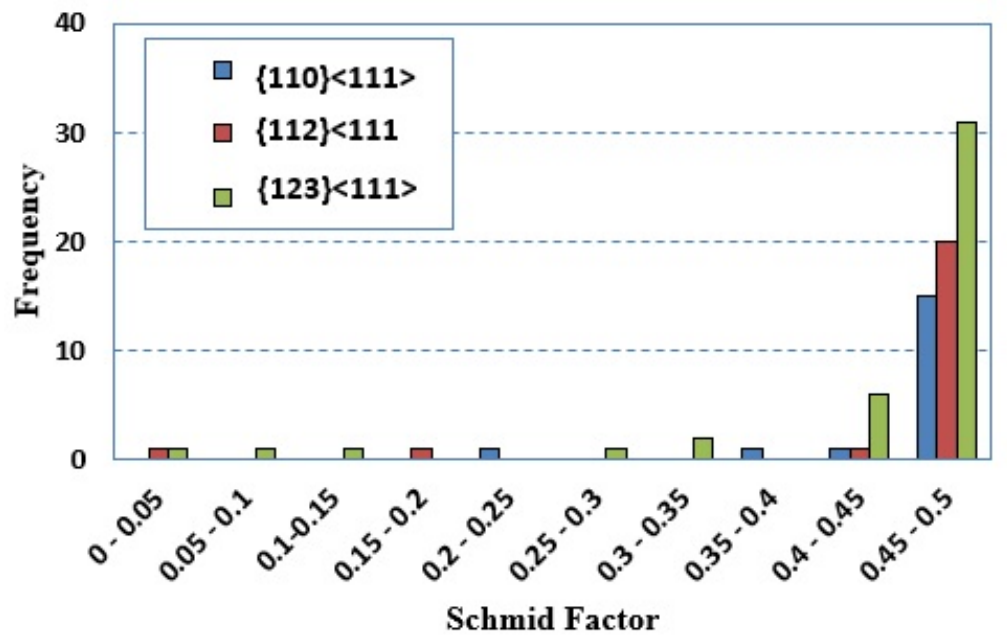

Figure 9. A histogram of Schmid factor distribution of slip systems for the TNTZ-0.7O sample deformed to $6.1 \%$ strain.

\section{Discussion}

The plastic deformation of the majority of $\beta$ Ti alloys is mainly attributed to the activation of slip systems, while twinning and martensite transformation have been observed in some $\beta$ Ti alloys [13]. Twinning and strain-induced-martensite (SIM) transformation are more likely in Ti alloys with a relatively unstable $\beta$ phase, which leads to a lower yield stress (YS) and a larger elongation-to-failure [14-17]. However, when the $\beta$ phase is stable, slip is the dominant deformation mechanism and this results in higher YS values [18-19]. Based on the acquired EBSD maps, the TNTZ-xO alloys exhibited a relatively weak texture. The results revealed that slip was the dominant plastic deformation mode for TNTZ-0.3O and TNTZ-0.7O, while twinning and slip were observed for TNTZ-0.1O. Two mechanisms are expected to be responsible for the increase in UTS with increasing O content:

i) The interstitial $\mathrm{O}$ atoms may occupy the octahedral or tetrahedral interstitial sites in the crystal lattice and thereby distort the lattice, which results in local stress fields that hinder the dislocation motion [20]. Higher O content results in a higher fraction of occupied sites and thus, more potential barriers for dislocation motion. 
ii) The solute $\mathrm{O}$ atoms may interact with screw and edge dislocations and impede the dislocation motion [21].

Twinning and slip were observed in TNTZ-0.1O. In our previous work, XRD and TEM investigations revealed that the SIM transformation, where the $\beta$ phase transforms to the orthorhombic ( $\alpha$ ") phase, occurs during plastic deformation in TNTZ0.10 [6]. The SIM transformation has been observed in other $\beta$ Ti alloys during mechanical loading, where during straining, the lattice of the $\beta$ unit cell became slightly larger in one direction and smaller in the other two directions, which resulted in the $\beta$ phase transforming to the $\alpha$ " phase [14-17]. Furthermore, low O content in Ti alloys enhances twinning [22, 23]. Fine slip traces were also observed in the SE SEM photomicrographs, see Figure 3. It is believed that the critical resolved shear stress for slip is greater than that for both twinning and SIM transformation, which consequently resulted in the activation of slip after the twinning and SIM transformation.

For TNTZ-0.3O and TNTZ-0.7O, only slip was observed, where the $\{110\}<123>$ exhibited the highest contribution. However, a previous study involving the normalized slip activity suggested that the high occurrence of $\{110\}<123>$ was likely due to the fact that the highest number of possible slip systems was associated with $\{110\}<123>$ compared with other two slip systems [12]. In another deformation study, twinning occurred in Ti-23Nb-0.7Ta-2Zr, while no twinning occurred in Ti-23Nb$0.7 \mathrm{Ta}-2 \mathrm{Zr}-1.2 \mathrm{O}$, where the martensite $\alpha$ " phase was suppressed by the addition of $\mathrm{O}$ during quenching [24]. $\mathrm{O}$ is usually considered to be an $\alpha$ stabilizer due to the fact that the $\beta$ transus temperature increases with increasing $O$ content [4]. In contrast, the presence of $O$ suppressed the formation of the $\alpha$ " and the $\omega$ phases, which suggests that $O$ behaves as a $\beta$ stabilizer in some $\beta$ Ti alloys [24-26]. In this work, the O addition enhanced the YS and also suppressed the SIM transformation and mechanical twinning during deformation, which suggests that $\mathrm{O}$ acts as a $\beta$ stabilizer in TNTZ-xO alloys.

Although, the TNTZ-0.7O UTS was higher than that for TNTZ-0.3O, the $\mathrm{e}_{\mathrm{f}}$ of TNTZ-0.7O was greater than that for TNTZ-0.3O [5,6]. In another $\beta$ Ti alloy (Ti-35Nb-7Zr-5Ta), the YS increased from $937 \mathrm{MPa}$ to $1081 \mathrm{MPa}$, when the O content increased from $0.46 \mathrm{wt} . \%$ to $0.68 \mathrm{wt} . \%$. [27]. However, no explanation was provided for that observation.

Some of the cracking for the TNTZ-0.3O sample initiated at a low strain of $\sim 2 \%$, which was early in the plastic deformation regime (see Figure 10). The cracks propagated throughout other grain boundaries at $\sim 3 \%$ strain. At $\sim 5 \%$ strain, the cracks appeared to widen. No new slip traces occurred within the grains at the crack edges, which was likely due to the fact that those areas were within the stress-free zone. On the other hand, no cracks were observed in the deformed TNTZ-0.7O. It is 
believed that the crack propagation and accumulation led to early fracture of the TNTZ-0.3O compared to the TNTZ-0.7O. Furthermore, the observed slip activity was higher and more homogenous in TNTZ-0.7O compared with TNTZ-0.3O.

The frequency of observed slip traces was relatively low in some areas of TNTZ-0.3O. Most of the cracks initiated in the regions where less slip traces were observed. Although this has not been confirmed, it is possible that this heterogeneous slip activity could be due to inhomogenous O distribution. Consequently, the lower slip activity in some regions could result in crack initiation to accommodate the local deformation in such areas. It is interesting to note that some of the TNTZ-0.3O cracks initiated at grain boundaries for grains close to hard orientations, i.e. $\{111\}$ oriented grains with respect to tensile direction.
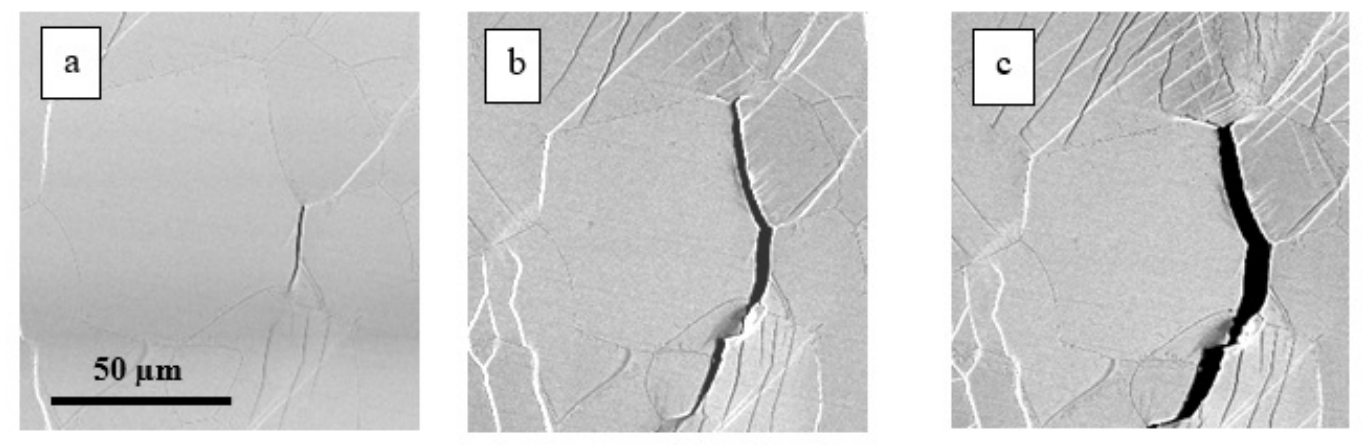

Figure 10. SE SEM photomicrographs of a deformed TNTZ-0.3O sample showing the evolution of a grain boundary crack: (a) $\sim 2 \%$ (b) $3 \%$ and (c) $5 \%$.

\section{$\underline{\text { 5. Conclusions }}$}

The effect of $\mathrm{O}$ content on the mechanical properties and deformation mode of TNTZ-xO alloys, where $\mathrm{x}=0.1,0.3$, or 0.7 , was investigated. The large elongation-to-failure exhibited by TNTZ-0.1O was explained to be a result of the activation and cooperation of three deformation mechanisms, i.e. SIM transformation ( $\beta$ phase to $\alpha$ " phase), $\{332\}<113>$ mechanical twinning, and the slip activity. Higher O contents suppressed both twinning and SIM transformation, and slip was the primary deformation mechanism for both TNTZ-0.3O and TNTZ-0.7O, where TNTZ-0.7O exhibited greater UTS and elongation-to-failure values. The in-situ SEM tensile tests revealed the initiation of intergranular cracks at the early stage of the plastic deformation in TNTZ0.3O. The crack propagation and accumulation are believed to have accelerated the fracture of the TNTZ-0.3O. On the other 
hand, significantly less cracking occurred in TNTZ-0.7O. In addition, SE SEM microphotographs indicated that the slip activity was more homogenous in TNTZ-0.7O.

\section{Acknowledgements}

This research was supported by the US Department of Energy, Office of Basic Energy Science through grant No. DE-FG0209ER46637. The authors are also grateful to Xin Cong of Hokoku Co. Ltd., Toyohashi, Japan and Ken Cho of Osaka University, Osaka, Japan for their assistance.

\section{References}

[1] M. Niinomi, Mater Sci Eng. A 243 (1998) 231-36.

[2] D. Kuroda et al., Mater Sci Eng. A 243 (1998) 244-49.

[3] T. Akahori et al., Mater Sci Eng. C 25 (2005) 248-54.

[4] H. Yilmazer et al, Mater Sci Eng. C 33 (2013) 2499-2507.

[5] F. Geng et al, Mater Sci Eng. A 528 (2011) 5435-45.

[6] H. Liu et al., Metall and Mat Trans A 48 (2017) 139-49.

[7] C.J. Boehlert et al., Phil Mag. 93 (2013) 598-617.

[8] H. Li, et al., Phil Mag. 92 (2012) 2923-46.

[9] H. Li, et al., Phil Mag. 93 (2013) 2875-95.

[10] H. Li et al., Phil Mag. 95 (2015) 691-729.

[11] V. Khademi et al., Proceedings of the 13th World Conference on Titanium, eds. Wiley-Blackwell (2016) 1109-

[12] V. Khademi, PhD Dissertation, Michigan State University, 2018.

[13] G. Lütjering and J.C. Williams, Titanium, eds. Berlin Heidelberg: Springer-Verlag (2003).

[14] R.J. Talling et al., Acta Mat. 57 (2009) 1188-98.

[15] H. Xing and J. Sun, Appl. Phy. Lett 93 (2008) 031908. 
[16] Y. Yang et al., Scr Mat. 58 (2008) 9-12.

[17] Y. Yang et al., Acta Mat. 58 (2010) 2778-87.

[18] S. Hanada and O. Izumi, Metall. Trans. A 18 (1987) 265-271.

[19] W. Liqiang et al., Rare Metal Mats Eng.38 (2009) 579-82.

[20] M. Tahara et al., Acta Materialia 59 (2011) 6208-18.

[21] E. Miura et al., Physica Status Solidi (A) 185 (2001) 357-72.

[22] S. Zaefferer, Mats Sci Eng. A 344 (2001) 20-30.

[23] E. Bertrand et al., Scr. Mater. 64 (2011) 1110-13.

[24] M. Besse et al., Acta Mater. 59 (2011) 5982-88.

[25] J.I. Qazi et al., JAI 2 (2005) 1-19.

[26] B.S. Hickman, J. Mater Sci. 4 (1969) 554-63.

[27] J.I. Qazi et al., JOM 56 (2004) 49-51. 